\title{
Generalized Lax Pairs, the Modified Classical Yang-Baxter Equation, and Affine Geometry of Lie Groups
}

\section{Martin Bordemann}

Fakultät für Physik der Universität Freiburg, Hermann-Herder-Strasse 3, W-7800 Freiburg i. Br., Federal Republic of Germany

\section{Received May 2, 1990}

Abstract. We generalize the usual Lax equation $\frac{d}{d t} L=[M, L]$ by $\frac{d}{d t} L=-\varrho(M) L$, where $\varrho$ is an arbitrary representation of a Lie algebra $g$ (the values of $M$ ) in a representation space $V$ (the values of $L$ ). The usual classical $r$-matrix programme for Hamiltonian integrable systems is generalized to $r$-matrices taking values in $g \otimes V$. The $r$-matrices are then considered as left invariant torsion-free covariant derivatives on a Lie group $K$ (with Lie algebra $V^{*}$ ). The Classical Yang-Baxter Equation (CYBE) is equivalent to the flatness of $K$ whereas the Modified CYBE implies that $K$ is an affine locally symmetric space. An example is discussed.

\section{Introduction}

In the last years, the interest in integrable Hamiltonian systems has rapidly grown because of the relation to the Classical Yang-Baxter Equations (CYBE) which in turn point towards the Quantum Yang-Baxter Equations (QYBE) and Quantum Groups. In various papers, e.g. by Sklyanin (cf. [1, 2]), Belavin and Drinfel'd (cf. $[3,4]$ ) and Semenov-Tyan-Shanskii (cf. [5]) classical $r$-matrices have been defined and discussed. Further algebraic properties and formulations of the CYBE and Poisson Lie groups using Schouten brackets are developed in the work of Kosmann-Schwarzbach and Magri (cf. [6]). In several articles (cf. [7, 8]) Babelon and Viallet studied the importance of classical $r$-matrices and the CYBE in the context of Hamiltonian systems defined by Lax pairs.

In this paper, an attempt is made to generalize the concept of Lax pairs by replacing the well-known Lax equation (cf. [9])

$$
\frac{d L}{d t}=[L, M]
$$

by

$$
\frac{d L}{d t}=-\varrho(M) L
$$


where $\varrho$ is an arbitrary representation of a Lie algebra $g$ in a representation space $V$, $M$ is a $\mathrm{g}$-valued function on phase space and $L$ is a $V$-valued function on phase space. For $V=\mathfrak{g}$ and $\varrho=$ ad (the adjoint representation of $\mathfrak{g}$ ) this reduces to the usual Lax equation. Such generalized Lax equations have also been considered in a more special context by Semenov-Tyan-Shanskii (cf. [10]) who uses restrictions of (co)adjoint representations and by Fomenko (cf. e.g. [11]) whose sectional operators are special $M$-functions depending linearly on $L$.

In Sect. 2 of this paper we shall give the definitions and apply the usual $r$-matrix programme (cf. e.g. [7]) to our more general situation: starting with the Lax pair we get $G$-invariant functions of $L$ as conservation laws ( $G$ being a $L i e$ group having Lie algebra g). Next, a more general $r$-matrix ansatz is formulated where the $r$-matrices are elements of $V \otimes \mathfrak{g}$ rather than $\mathfrak{g} \otimes \mathfrak{g}$. This guarantees Poisson commutativity of the above conservation laws. We shall then give a more general form of the (Modified) CYBE which serve as a sufficient condition to the consistency of a Lie structure on $\mathfrak{f}=V^{*}$ defined by the $r$-matrices.

In Sect. 3 of this paper we shall adopt the point of view of affine geometry rather than the usual symplectic geometry: We shall interpret the generalized $r$-matrices as left invariant affine torsion-free connections $\nabla$ on a Lie group $K$ (having Lie algebra $\left.\mathfrak{f}=V^{*}\right)$ and define the Lie algebra $\mathfrak{g}$ to be any subalgebra of $\mathrm{gl}(\mathfrak{f})$ containing (up to projections) the Lie algebra generated by all covariant derivatives at the identity along left invariant vector fields on $K$. A special case of this, namely the canonical "half commutator" connection on a semisimple Lie group appears in two papers of Ferreira (cf. [12]). The Jacobi identity for the Lie structure on $\mathfrak{f}^{\text {is }}$ then reflected in the first Bianchi identity of the Riemann curvature tensor of $\nabla$. We shall show that this construction will give rise to new families of Poisson commuting conservation laws. The CYBE and the Modified CYBE will then appear as special cases of this, i.e. zero curvature for CYBE whereas the Modified CYBE will correspond to a covariantly constant curvature tensor thus giving the Lie group $K$ the structure of an (in general noncanonical) affine locally symmetric space. Here the crucial condition will turn out to be the possibility of twilling together (in the sense of Kosmann-Schwarzbach and Magri, cf. [6]) the two Lie algebras $\mathfrak{g}$ and $\mathfrak{f}$ to a larger one defined on the direct vector space sum $\mathfrak{g} \oplus \mathfrak{f}$ (which contain the Manin triple algebras $\mathfrak{g} \oplus \mathfrak{g}^{*}$ as a special case). We shall show that this twilled extension is a symmetric Lie algebra of infinitesimal affine transformations on the affine symmetric space $K$.

Section 4 of this letter contains an example for a generalized Lax pair, namely the now classical left invariant geodesic motion on a Lie group $K$ (see e.g. the work of Fomenko and Mishchenko [18]): here, one half of the equations of motion has the usual coadjoint Lax form but we show that it can be rewritten in a second Lax form not containing the coadjoint representation thus giving rise to more Poisson commuting conservation laws.

In this paper we do not discuss the difficult problem of completeness of these conservation laws and the possibly prominent rôle of the (co)adjoint representation in this context.

\section{Generalized Lax Pairs, $r$-Matrices and the (M)CYBE}

Let $(P, \omega)$ be a symplectic manifold (a phase space) and $H: P \rightarrow \mathbb{R}$ a fixed realvalued smooth function (a Hamiltonian). We refer to $(P, \omega, H)$ as a Hamiltonian 
system, $X_{H}=\omega^{\#} d H$ is the Hamiltonian vector field of $H$, and the Hamiltonian equations are $\dot{p}=X_{H}(p)$. Recall that $(P, \omega, H)$ is completely integrable (in the sense of Liouville) iff $H$ is a function of $n(=1 / 2 \operatorname{dim} P)$ functionally independent, Poisson commuting conservation laws $F_{i}: P \rightarrow \mathbb{R}(1 \leqq i \leqq n)$ (see the books of Abraham and Marsden ([13], where we take our sign conventions from) or Fomenko and Trofimov [11] for details). The following procedure will serve as an ansatz to get completely integrable Hamiltonian systems.

2.1. Definition. We call a quintuple $(\mathfrak{g}, \varrho, V, L, M)$ a (generalized) Lax pair for a Hamiltonian system $(P, \omega, H)$ iff

i) $\mathfrak{g}$ is a finite dimensional real Lie algebra,

ii) $V$ is a finite dimensional real vector space,

iii) $\varrho: \mathfrak{g} \rightarrow \operatorname{Hom}(V, V)$ is a representation of $\mathfrak{g}$ in $V$,

iv) $L: P \rightarrow V$ is a smooth map and

v) $M: P \rightarrow \mathfrak{g}$ is a smooth map such that

$$
d L(p) X_{H}(p)=-\varrho(M(p)) L(p) \text { for all } p \text { in } P .
$$

On the solution curves of $X_{H}$ equation (*) is by the chain rule equivalent to

$$
\frac{d L}{d t}=-\varrho(M) L .
$$

Of course, for $\mathfrak{g}=V$ and $\varrho=a d$ this reduces to the usual Lax equation. We say that the Hamiltonian system $(P, \omega, H)$ is defined by $(\mathfrak{g}, \varrho, V, L, M)$ iff $d L(p)$ is injective for all $p$ in $P$ because then the inhomogeneous linear equation $(*)$ is uniquely solvable for $X_{H}$ if solvable at all. $P$ can then be considered as an immersed submanifold of $V$.

In numerous cases $M$ is a function of $L$ which implies that Eq. (2.2) defines a differential equation on $V$ that can be investigated without referring to $(P, \omega, H)$.

Let $G$ be a connected Lie group having Lie algebra $g$ such that $\varrho$ exponentiates to a representation of $G$ in $V$ which we shall also call $\varrho$. Recall that the $G$-orbit $G \cdot v$ through $v \in V$ is the set $\{\varrho(g) v \mid g \in G\}$. Now, since the tangent space $T_{v}(G \cdot v)$ is given by the space $\{\varrho(\xi) v \mid \xi \in \mathfrak{g}\}$ the geometric content of Eq. $(*)$ is the easily seen fact that $L$ maps the integral curves of $X_{H}$ onto curves in $V$ that are always constrained to some $G$-orbit in $V$. Hence the following proposition is an immediate consequence:

2.3. Proposition. Let $(\mathfrak{g}, \varrho, V, L, M)$ be a generalized Lax pair for a Hamiltonian system $(P, \omega, H)$. Furthermore, let $f: V \rightarrow \mathbb{R}$ be a G-invariant smooth function, i.e. $f(\varrho(g) v)=f(v)$ for all $g \in G$ and $v \in V$.

Then $f \circ L$ is a conservation law, i.e.

$$
\frac{d(f \circ L)}{d t}=\{f \circ L, H\}=0 .
$$

Proof. $G$-invariant functions are always constant on each $G$-orbit, and the equation follows from the infinitesimal $G$-invariance of $f$ :

$$
d f(v) \varrho(\xi) v=0 \quad \text { for all } \xi \in \mathfrak{g} \text { and } v \in V .
$$

Hence

$$
\frac{d}{d t}(f \circ L)=d f(L) \frac{d}{d t} L=-d f(L) \varrho(M) L=0 .
$$


For the usual Lax pairs, $f$ is mostly taken to be a trace polynomial trace $\left(L^{k}\right)(k$ a positive integer) which is invariant under conjugation $L \mapsto g L g^{-1}$ (that is the adjoint representation of $G$ in $\mathfrak{g}=V$ ).

So far, the Hamiltonian structure of the phase space $P$ did not enter and everything mentioned above is also valid for arbitrary vector fields on $P$. The next step in the programme is the question: when do these conservation laws $f \circ L$ Poisson commute among themselves?

Let $e_{i}(1 \leqq i \leqq \operatorname{dim} V)$ be a basis of $V$ and $T_{A}(1 \leqq A \leqq \operatorname{dimg})$ be a basis of $\mathfrak{g}$. Then for $v=v^{i} e_{i} \in V$ and $\xi=\xi^{A} T_{A} \in \mathrm{g}$ one has the "matrix elements" $\varrho_{A}{ }^{i}$ of the representation $\varrho$, i.e. $\xi^{A} v^{j} \varrho_{A}{ }^{i}=(\varrho(\xi) v)^{i}$ (for $V=\mathfrak{g}, \varrho=a d$ these matrix elements are nothing but the structure constants of the Lie algebra g). Now the Poisson bracket $\{f \circ L, h \circ L\}$ for arbitrary functions $f, h: V \rightarrow \mathbb{R}$ is easily calculated:

$$
\{f \circ L, h \circ L\}=\frac{\partial f}{\partial v^{i}} \circ L \frac{\partial h}{\partial v^{j}} \circ\left\{L^{i}, L^{j}\right\} .
$$

If both $f$ and $h$ are $G$-invariant, then the above Poisson bracket will vanish if the Poisson bracket $\left\{L^{i}, L^{j}\right\}$ "somehow contains the representation $\varrho$ such that the infinitesimal $G$-invariance [Eq. (2.4)] can be applied." To be more precise we consider two smooth maps which we shall call classical r-matrices in the sequel

$$
r_{+}, r_{-}: V \times P \rightarrow V \otimes \mathfrak{g}
$$

and make the following generalized r-matrix ansatz:

$$
\left\{L^{i}, L^{j}\right\}(p)=-r_{+}{ }^{i A}(L(p), p) \varrho_{A}{ }^{j}{ }_{k} L^{k}(p)+r_{-}{ }^{j A}(L(p), p) \varrho_{A k}{ }_{k} L^{k}(p) .
$$

As a consequence we have the

2.8. Theorem. Let $(\mathfrak{g}, \varrho, V, L, M)$ be a generalized Lax pair for the Hamiltonian system $(P, \omega, H)$ allowing for classical $r$-matrices that obey Eq. 2.7. Then for two real-valued G-invariant functions $f$ and $h$ on $V$ the Poisson bracket $\{f \circ L, h \circ L\}$ vanishes.

Proof. Using Eq. (2.5) and suppressing the arguments of $r_{+}$and $r_{-}$we get

$$
\{f \circ L, h \circ L\}=-\frac{\partial f}{\partial v^{i}} \circ L r_{+}{ }^{i A} \frac{\partial h}{\partial v^{j}} \circ L \varrho_{A}{ }^{j}{ }_{k} L^{k}+\frac{\partial f}{\partial v^{i}} \circ L \varrho_{A}{ }^{i} L^{k} \frac{\partial h}{\partial v^{j}} \circ L r_{-}{ }^{j A}
$$

and the underlined terms both give zero because of infinitesimal $G$-invariance [Eq. (2.4)].

Again, for $V=\mathfrak{g}$ and $\varrho=$ ad the classical $r$-matrices take values in $\mathfrak{g} \otimes \mathfrak{g}$, and the above theorem specializes to the fact proven, e.g. in [7] that arbitrary trace polynomials of $L$ Poisson commute among themselves.

Making an ansatz like Eq. (2.7) requires a consistency check because on the left-hand side is a Lie bracket coming from the Poisson structure that satisfies antisymmetry and the Jacobi identity. Hence the classical $r$-matrices have to obey some consistency conditions. In the following, we shall only consider constant $r$-matrices (for $L$-dependent $r$-matrices in the usual approach see [8]). This implies that the component functions $L^{i}$ close to a finite dimensional Lie subalgebra $\mathfrak{f}_{L}$ of the Poisson algebra of functions on the phase space $(P, \omega)$ since the right-hand side of Eq. (2.7) is then linear in L. Finite dimensional Lie subalgebras of Poisson algebras have extensively been studied by Mishchenko and Fomenko (cf. [11, 14]). 
In case $\mathfrak{f}_{L}$ exponentiates to a global canonical action of some connected Lie group $K_{L}$ (having Lie algebra $\mathfrak{f}_{L}$ ) on phase space $L$ will be an $\mathrm{Ad}^{*}$-equivariant momentum map (see [13] for details). Now the values of $L$ will in general span only a subspace $V_{L}$ of $V$ which need not be $G$-invariant (the $L$ for the $s l(n, \mathbb{R})$ Toda chain occupies only diagonal and first upper and lower off-diagonal matrix elements, see e.g. [7]). Therefore the conditions for the classical $r$-matrices will in general depend on the specific $L$. But if one is interested in the investigation of all generalized Lax pairs having the same phase space $(P, \omega)$, the same $\mathrm{g}, \varrho$, and $V$ and the same classical $r$-matrices $r_{+}$and $r_{-}$then one has to impose the stronger condition that Eq. (2.7) ought to be consistent with the Poisson structure of all such $L$ 's. This stronger condition then says that

$$
f^{i j}{ }_{k} \equiv-r_{+}{ }^{i A} \varrho_{A}{ }^{j}{ }_{k}+r_{-}{ }^{j A} \varrho_{A}{ }^{i}{ }_{k}
$$

should be the structure constants of a Lie structure on $V^{*}$, the dual space of $V$. We shall now come to an index-free notation: we set $\mathfrak{f} \equiv V^{*}$ and consider $r_{+}$and $r_{-}$as linear maps $\mathfrak{f} \rightarrow \mathfrak{g}: x=x_{i} e^{i} \mapsto r_{ \pm}(x) \equiv x_{i} r_{ \pm}{ }^{i A} T_{A}$, where $e^{i}$ are dual basis vectors to $e_{i}$. This construction is in fact canonical: $V \otimes \mathfrak{g} \sim \mathfrak{f}^{*} \otimes \mathfrak{g} \sim \operatorname{Hom}(\mathfrak{f}, \mathfrak{g})$. The representation $\varrho$ dualizes to a representation $\varrho^{*}: \mathfrak{g} \rightarrow \operatorname{Hom}(\mathfrak{f}, \mathfrak{f})$ in the usual way: $\left(\varrho^{*}(\xi) x\right)(v)$ $\equiv-x(\varrho(\xi) v)$ for all $x \in \mathfrak{F}, \xi \in \mathfrak{g}$ and $v \in V$. We shall write $\xi \cdot x$ for $\varrho^{*}(\xi) x$.

With these abbreviations condition (2.9) can be reformulated as follows:

$$
[x, y]_{R} \equiv r_{+}(x) \cdot y-r_{-}(y) \cdot x
$$

should define a Lie bracket for all $x, y \in \mathfrak{f}$. With

$$
r \equiv 1 / 2\left(r_{+}+r_{-}\right), \quad c \equiv 1 / 2\left(r_{+}-r_{-}\right)
$$

we have the following

2.12. Proposition. Equation (2.10) defines a Lie bracket on $\mathfrak{f}$ iff for all $x, y, z \in \mathfrak{f}$

i) $[x, y]_{R}=r(x) \cdot y-r(y) \cdot x \Leftrightarrow c(x) \cdot y+c(y) \cdot x=0$,

ii) $\left([r(x), r(y)]-r\left([x, y]_{R}\right)\right) \cdot z+$ cycl. $=0$.

Proof. The antisymmetry condition i) is obvious. In order to prove the Jacobi identity we write out the terms

$$
\begin{aligned}
& {\left[[x, y]_{R}, z\right]_{R}=\mathbf{r}\left([\mathbf{x}, \mathbf{y}]_{\mathbf{R}}\right) \cdot \mathbf{z}-r(z) \cdot(r(x) \cdot y)+r(z) \cdot(r(y) \cdot x)} \\
& {\left[[z, x]_{R}, y\right]_{R}=r\left([z, x]_{R}\right) \cdot y-r(y) \cdot(r(z) \cdot x)+\mathbf{r}(\mathbf{y}) \cdot(\mathbf{r}(\mathbf{x}) \cdot \mathbf{z})} \\
& {\left[[y, z]_{R}, x\right]_{R}=r\left([y, z]_{R}\right) \cdot x-\mathbf{r}(\mathbf{x}) \cdot(\mathbf{r}(\mathbf{y}) \cdot \mathbf{z})+r(x) \cdot(r(z) \cdot y)}
\end{aligned}
$$

and use the following representation identity: $[\xi, \eta] \cdot x=\xi \cdot(\eta \cdot x)-\eta \cdot(\xi \cdot x)$ for all $\xi, \eta \in \mathfrak{g}$ and $x \in \mathfrak{f}$ to re-arrange terms in the cyclic sum. For instance, the sum of the terms in yield the term written out in ii) above.

Note that for a specific Lax pair $(\mathfrak{g}, \varrho, V, L, M)$ of a Hamiltonian $\operatorname{system}(P, \omega, H)$ allowing for classical $r$-matrices that obey $2.12 \mathrm{~L}$ defines a Lie homomorphism

$$
L: \mathfrak{f} \rightarrow \mathfrak{f}_{L}: x \mapsto\langle L, x\rangle \equiv x_{i} L^{i}
$$

(using the natural pairing $\langle$,$\rangle of V$ and $V^{*}$ ) since Eqs. (2.7) and (2.10) together yield the following:

$$
\{\langle L, x\rangle,\langle L, y\rangle\}=\left\langle L,[x, y]_{R}\right\rangle \text { for all } x, y \in \mathfrak{F} .
$$


Furthermore, we can directly transfer a proposition in [8, Part 3], namely that each Hamiltonian of the special form $f \circ L$ with $G$-invariant $f: V \rightarrow \mathbb{R}$ admits the following Lax pair $(\mathfrak{g}, \varrho, V, L,-r(d f(L)))$ :

$$
\frac{d L}{d t}=\{L, f \circ L\}=\varrho(r(d f(L)) L
$$

since the first summand in the Poisson bracket vanishes by infinitesimal $G$-invariance of $f$ [cf. Eq. (2.4)].

Now we return to Proposition 2.12. Recall that the crucial condition for the Jacobi identity was the vanishing of the cyclic sum over

$$
([r(x), r(y)]-r(r(x) \cdot y)+r(r(y) \cdot x)) \cdot z .
$$

An obvious ansatz satisfying Eq. 2.12 ii) would be

$$
[r(x), r(y)]-r\left([x, y]_{R}\right)=0 \text { for all } x, y \in \mathfrak{f}
$$

which we call the Classical Yang-Baxter Equation (CYBE) for $r$. This is of course equivalent to $r: \mathfrak{f} \rightarrow \mathfrak{g}$ defining a homomorphism of Lie algebras.

A more sophisticated ansatz is obtained if the 0 on the r.h.s. of Eq. (2.17) is replaced by a term containing the map $c$ [cf. (2.11)]:

2.18. Theorem. The notations being explained above let the map $c$ satisfy

a) $c(x) \cdot y+c(y) \cdot x=0$ for all $x, y \in \mathfrak{f}$ (antisymmetry)

b) $c(\xi \cdot x)=[\xi, c(x)]$ for all $x \in \mathfrak{f}$ and $\xi \in \mathfrak{g}$ (G-invariance).

Then the following two conditions are equivalent and imply the Jacobi identity for the bracket $[,]_{R}$ on $\mathfrak{\mathrm { f }}$ :

i) $[r(x), r(y)]-r(r(x) \cdot y)+r(r(y) \cdot x)=-[c(x), c(y)] \quad \forall x, y \in \mathfrak{f}$ (Modified Classical Yang-Baxter Equation (MCYBE) for $r$ ),

ii) $\left[r_{ \pm}(x), r_{ \pm}(y)\right]-r_{ \pm}\left([x, y]_{R}\right)=0 \forall x, y \in \mathfrak{f}$

(Classical Yang-Baxter Equation (CYBE) for $r_{+}$and $r_{-}$).

Proof. Note that $r_{ \pm}=r \pm c$, and the equivalence of i) and ii) follows in the following manner:

$$
\begin{aligned}
& {[r(x) \pm c(x), r(y) \pm c(y)]-r\left([x, y]_{R}\right) \mp c(r(x) \cdot y) \pm c(r(y) \cdot x)} \\
& =[r(x), r(y)]-r\left([x, y]_{R}\right)+[c(x), c(y)] \\
& \quad \pm([r(x), c(y)]-[r(y), c(x)]-c(r(x) \cdot y)+c(r(y) \cdot x)),
\end{aligned}
$$

and the \pm -term vanishes by the $G$-invariance of $c$. Since for all $x, y, z \in \mathfrak{f}$

$$
\begin{aligned}
{[c(x), c(y)] \cdot z } & =c(x) \cdot(c(y) \cdot z)-c(y) \cdot(c(x) \cdot z) \\
& =-c(c(y) \cdot z) \cdot x-c(c(z) \cdot x) \cdot y \text { (by antisymmetry) } \\
& =-[c(y), c(z)] \cdot x-[c(z) \cdot c(x)] \cdot y \text { (by } G \text {-invariance) }
\end{aligned}
$$

the Jacobi identity holds.

Here, the map $r$ is in general no longer a Lie homomorphism, but the two $r$-matrices $r_{+}$and $r_{-}$both are. As a justification of the name "(M)CYBE" and a motivation to consider $G$-invariance (property b) of c) we shall discuss two wellknown cases: 
1. $\varrho=\mathrm{ad}^{*}, V=\mathrm{g}^{*}:$ Here $\mathfrak{f}$ is equal to $\mathrm{g}$ as a vector space and $\varrho^{*}=\mathrm{ad}$. Fix a linear map $R: \mathfrak{g} \rightarrow \mathfrak{g}$, set $r=R$ and $c=1$. Obviously, $c$ satisfies conditions a) and b) of the above theorem. The bracket $[,]_{R}$ on $\mathfrak{f}=\mathfrak{g}$ yields a second Lie structure on $\mathfrak{g}$ :

$$
[x, y]_{R}=[R x, y]+[x, R y] \text { for all } x, y \in \mathfrak{g}=\mathfrak{f},
$$

and the MCYBE as defined above take the familiar form

$$
[R x, R y]-R[R x, y]-R[x, R y]=-[x, y] .
$$

For the equivalence of the MCYBE with the CYBE for $R_{ \pm} \equiv R \pm 1$ see [5] or [7]. Lie algebras $g$ with an $r$-matrix $R$ satisfying the MCYBE are sometimes called Baxter Lie algebras (cf. [7]). Semisimple Baxter Lie algebras with $r$-matrices $R$ that are antisymmetric with respect to the Killing form of $g$ have been classified by Belavin and Drinfel'd (cf. [3]).

2. $\varrho=$ ad, $V=\mathfrak{g}$ : Here $\mathfrak{f}=\mathrm{g}^{*}$ and $\varrho^{*}=\mathrm{ad}^{*}$. In an abuse of notation, fix a linear map $r: \mathrm{g}^{*} \rightarrow \mathrm{g}$ (which can canonically be considered as an element of $\mathrm{g} \otimes \mathrm{g}$ ) and set $r_{+}=r$ and $r_{-}=-r^{*}$ (which simply amounts to the negative of the transposition of the matrix $r)$. Hence $c=1 / 2\left(r+r^{*}\right)$ is self-dual: $c^{*}=c$. Therefore we have the following equation for all $x, y \in \mathfrak{g}^{*}$ and $\xi \in \mathfrak{g}$ :

$$
(c(x) \cdot y)(\xi)=y([\xi, c(x)])=x\left(c^{*}\left(\operatorname{ad}(\xi)^{*} y\right)\right)=-x(c(\xi \cdot y)) .
$$

This clearly shows the equivalence of antisymmetry (condition a) in 2.18) and $G$-invariance (condition b) in 2.18) for $c$ in this particular case.

Now, the right-hand side of the CYBE as defined above reads for $x, y, z \in \mathrm{g}^{*}$ and $\gamma_{j k}^{i}(1 \leqq i, j, k \leqq \operatorname{dim} \mathrm{g})$ denoting the structure constants of $\mathrm{g}$ :

$$
\begin{aligned}
z([ & \left.\left.r_{+}(x), r_{+}(y)\right]-r_{+}\left([x, y]_{R}\right)\right) \\
= & z([r(x), r(y)])-z\left(r\left(\operatorname{ad}^{*}(r(x)) y\right)\right)-z\left(r\left(\operatorname{ad}^{*}\left(r^{*}(y)\right) x\right)\right) \\
= & z([r(x), r(y)])-y\left(\left[r^{*}(z), r(x)\right]\right)-x\left(\left[r^{*}(z), r^{*}(y)\right]\right) \\
= & x_{i} y_{j} z_{k}\left(r^{i s} r^{j t} \gamma^{k}{ }_{s t}-r^{s k} r^{i t} \gamma_{s t}^{j}-r^{s k} r^{t j} \gamma_{s t}^{i}\right) \\
= & x \otimes y \otimes z\left(r^{i s} r^{j t} T_{i} \otimes T_{j} \otimes\left[T_{s}, T_{t}\right]-r^{s k} r^{i t} T_{i} \otimes\left[T_{s}, T_{t}\right] \otimes T_{k}\right. \\
& \left.-r^{s k} r^{t j}\left[T_{s}, T_{t}\right] \otimes T_{j} \otimes T_{k}\right) \\
= & x \otimes y \otimes z\left(\left[r_{13}, r_{23}\right]+\left[r_{12}, r_{23}\right]+\left[r_{12}, r_{13}\right]\right),
\end{aligned}
$$

where $r_{12} \equiv r^{i t} T_{i} \otimes T_{t} \otimes 1, r_{13} \equiv r^{s k} T_{s} \otimes 1 \otimes T_{k}, r_{23} \equiv r^{j t} 1 \otimes T_{j} \otimes T_{t}, 1$ being the identity in the universal enveloping algebra of $\mathfrak{g}$. Hence the original version of the CYBE (cf. [3] or [5])

$$
\left[r_{12}, r_{13}\right]+\left[r_{12}, r_{23}\right]+\left[r_{13}, r_{23}\right]=0
$$

is also recovered in this framework. In the beginning, only antisymmetric $r$ (in the sense that $c=0 \Leftrightarrow r_{12}=-r_{21}$ and not in the more general sense of condition $2.18 \mathrm{a}$ )) had been discussed (cf. [4]) giving $\mathfrak{g}$ the structure of a so called Lie bialgebra.

We remark that for semisimple $g$ a classification of the solutions of the MCYBE in the sense of Theorem 2.18 can partially be done by using the fact that the kernel of the map $c$ is a $\mathfrak{g}$-invariant subalgebra of $\mathfrak{f}$ and the image of $c$ is an ideal of $\mathfrak{g}$. We shall do this in another paper. 


\section{3. (Modified) Classical Yang-Baxter Equations and Affine Geometry on Lie Groups}

In the previous part we were starting with a given (generalized) Lax pair, i.e. a given Lie algebra $g$, a given representation $\varrho$ and a given representation space $V$ and successively constructed a Lie structure on $\mathfrak{f}$, the dual space of the representation space. In this part, we want to reverse this procedure:

Given a (simply) connected Lie group $K$ having Lie algebra $\mathfrak{f}$, how can we construct the Lie algebra $g$, the representation $\varrho$ and possibly a typical Lax pair $(L, M)$ ?

Now, the Lie algebra $\varrho^{*}(\mathfrak{g})$ is a subalgebra of the Lie algebra $\mathfrak{g l}(\mathfrak{f})$ of all linear maps $\mathfrak{f} \rightarrow \mathfrak{f}$. According to Proposition 2.12 we are looking for linear maps $r$. $\equiv \varrho^{*}(r()): \mathfrak{f} \rightarrow \varrho^{*}(\mathfrak{g}) \subset \mathfrak{g l}(\mathfrak{f})$ ( $\mathfrak{g}$ being yet unspecified) such that the now fixed Lie bracket $[,]_{R}$ on $\mathfrak{f}$ can be expressed by

$$
[x, y]_{R}=r(x) \cdot y-r(y) \cdot x \text { for all } x, y \in \mathfrak{F} .
$$

The idea is now to interpret $[,]_{R}$ as a Lie bracket $[$,$] of vector fields, r($ ). as a covariant derivative $\nabla$, and the above equation as a vanishing torsion condition. More precisely, let $\hat{x}, \hat{y}$ denote the left invariant vector fields generated by $x, y \in \mathfrak{f}$ (i.e. $\hat{x}(k) \equiv T_{e} L_{k} x$ for $k \in K, e \equiv$ identity element of $K, L_{k} k^{\prime} \equiv k k^{\prime}$ left multiplication, $\hat{x}(k)=k x$ for matrix groups). By definition $[\hat{x}, \hat{y}]=\left([x, y]_{R}\right)^{\hat{y}}$. Furthermore, let $\nabla$ be a left invariant, torsion-free covariant derivative on $K$ : then we have the following vanishing torsion equation:

$$
[\hat{x}, \hat{y}]=\nabla_{\hat{x}} \hat{y}-\nabla_{\hat{y}} \hat{x} \quad \text { for all } x, y \in \mathfrak{H} .
$$

Since the vector field $\nabla_{\hat{x}} \hat{y}$ is again left invariant it is determined by its value at the identity and we can define a linear map $r .: \mathfrak{f} \rightarrow \mathfrak{g l}(\mathfrak{f})$ by

$$
r(x) \cdot y \equiv \nabla_{\hat{x}} \hat{y}(e) \text { for all } x, y \in \mathfrak{f},
$$

where at the moment the dot is yet unspecified and will be interpreted in a second step. On the other hand, since the left invariant vector fields form a basis for all vector fields on $K$ any left invariant $\nabla$ is determined by its values on the left invariant vector fields. Hence, an arbitrary linear map $r .: \mathfrak{f} \rightarrow \mathfrak{g l}(\mathfrak{f})$ defines a unique left invariant $\nabla$ by means of the above equation (for details of this procedure see [15], where $r(x)$. is denoted by $\Lambda(x) y)$. Moreover any left invariant torsion-free $\nabla$ is defined by an $r$. of the following special form:

$$
r(x) \cdot y=1 / 2[x, y]_{R}+U(x, y) \text { for all } x, y \in \mathfrak{f},
$$

where $U$ is any symmetric bilinear map $\mathfrak{F} \times \mathfrak{f} \rightarrow$ (see [15, Chap. X, p. 192] for a proof). In case $U$ vanishes $\nabla$ is the canonical torsion-free ("half commutator") connection which has been used e.g. by Ferreira [12].

In order to define the Lie algebra $g$, the representation $\varrho^{*}$, and the $r$-matrices (without dot) let $\mathfrak{a}_{\mathfrak{f}}$ be the Lie subalgebra of $\mathfrak{g l}(\mathfrak{t})$ generated by all $r(x)$., $x \in \mathfrak{f}$ :

$$
\mathfrak{a}_{\mathfrak{f}} \equiv \mathbb{R}-\operatorname{span}\{r(x) .,[r(x) ., r(y) \cdot],[[r(x) ., r(y) .], r(z) .] \text {, etc. } \mid x, y, z \in \mathfrak{f}\} .
$$

Choose any subalgebra $\hat{\mathfrak{g}}$ of $\mathfrak{g l}(\mathfrak{f})$ containing $\mathfrak{a}_{\mathfrak{f}}$, take any Lie algebra $\mathfrak{g}$ such that there is a surjective homomorphism $\varrho^{*}: \mathfrak{g} \rightarrow \hat{\mathfrak{g}}$, and find a linear map $r: \mathfrak{f} \rightarrow \mathfrak{g}$ such that $r(x) .=\varrho^{*}(r(x))$. Defining $V \equiv \mathfrak{f}^{*}$ and $\varrho \equiv\left(\varrho^{*}\right)^{*}$ we clearly obtain the structure we wanted. For instance, a natural choice would be $\mathfrak{g}=\hat{\mathfrak{g}}=\mathfrak{a}_{\mathfrak{t}}, \varrho^{*}=$ identity on $\mathfrak{a}_{\mathfrak{i}}$, and $r=r$. 
Now, we get a very natural interpretation for the left-hand side of the (M)CYBE:

$$
\begin{aligned}
\left([r(x), r(y)]-r\left([x, y]_{R}\right)\right) \cdot z & =\left(\nabla_{\hat{x}} \nabla_{\hat{y}}-\nabla_{\hat{y}} \nabla_{\hat{x}}-\nabla_{[\hat{x}, \hat{y}]}\right) \hat{z}(e) \\
& =R(\hat{x}, \hat{y}) \hat{z}(e),
\end{aligned}
$$

where $R$ denotes the Riemann curvature tensor of $\nabla$. Therefore we can re-interpret the Jacobi identity condition in Proposition 2.12 ii) as the first Bianchi identity for a torsion-free curvature tensor (cf. e.g. [15, Chap. III, p. 135]). If we also take into account Proposition 2.3, Theorem 2.8, and Proposition 2.12 we have the following

3.6. Theorem. Let $K$ be a finite-dimensional real Lie group having Lie algebra $\mathfrak{f}$ and let $\nabla$ be a left invariant torsion-free connection on $K$ defined by a linear map $r .:$ $\rightarrow \mathfrak{g l}(\mathfrak{f})$ as explained above. Choose $\left(\mathfrak{g}, \varrho, V=\mathfrak{l}^{*}\right)$ in the above manner. Assume that there is a Hamiltonian system $(P, \omega, H)$ admitting a generalized Lax pair $(\mathfrak{g}, \varrho, V, L, M)$ such that Eqs. 2.14 (i.e. $\left.\{\langle L, x\rangle,\langle L, y\rangle\}=\left\langle L,[x, y]_{R}\right\rangle\right)$ is satisfied for all $x, y \in \mathfrak{F}$. Then all the $G$-invariant functions of L are conservation laws and Poisson commute among themselves.

Note that the vanishing torsion is the only condition $\nabla$ has to satisfy. An example for a Hamiltonian system satisfying the hypothesis of this theorem will be given in Sect. 4.

We shall now investigate the specific affine connections $\nabla$ underlying the CYBE and the MCYBE as defined in the preceding part:

The CYBE for $r$ [cf. Eq. (2.17)] simply means that the curvature of the corresponding $\nabla$ is zero, i.e. that $K$ is flat. For an account of left invariant flat torsion-free connections on a Lie group $K$ see the work of Medina [16]. We shall derive the differential geometric meaning of the MCYBE (in the general sense of Theorem 2.18) in the following

3.7. Theorem. In the notation of Theorem 2.18 the curvature tensor of the MCYBE

$$
R_{e}(x, y) z \equiv-[c(x), c(y)] \cdot z \text { for all } x, y, z \in \mathfrak{f}
$$

is $\mathfrak{g}$-invariant, i.e. for all $x, y, z \in \mathfrak{F}$ and $\xi \in \mathfrak{g}$,

$$
\xi \cdot R_{e}(x, y) z-R_{e}(x, y) \xi \cdot z-R_{e}(\xi \cdot x, y) z-R_{e}(x, \xi \cdot y) z=0 .
$$

In particular, setting $\xi=r(w), w \in \mathfrak{f}$, this means that the curvature tensor is covariantly constant which in turn is equivalent to the Lie group $K$ being an affine locally symmetric space.

Proof. The first statement easily follows from the $G$-invariance of $c$. Since $r(w)$ represents $\nabla_{\hat{w}}$ at the identity it follows that the tensor field $\nabla R$ vanishes. But this fact together with the assumed vanishing torsion of $\nabla$ is equivalent to $K$ being locally symmetric (cf. [15, Chap. XI, p. 222]).

An affine (locally) symmetric space is also characterized by the condition that around each point the (local) parity transformation reflecting geodesics of $\nabla$ is a (locally) affine transformation (cf. [15, Chap. XI, p. 222]). If $K$ is simply connected and complete in the sense that the affine parameter of each geodesic is defined on the whole real line, then $K$ will be globally affine symmetric (cf. again [15, Chap. XI]).

Note that on every Lie group $K$ there exists a canonical left and right invariant affine symmetric structure which is given by the standard reflections $s_{k^{\prime}}(k)$ 
$\equiv k^{\prime} k^{-1} k^{\prime}\left(k, k^{\prime} \in K\right)$ and the 0 -connection $\nabla_{\hat{x}} \hat{y} \equiv 1 / 2[x, y]$. Here the geodesics of $\nabla$ are always equal to shifted one-parameter-subgroups of the form $\tau \mapsto k \exp (\tau x)$, $k \in K, x \in \mathfrak{f}, \tau \in \mathbb{R}$ (cf. [15, Chap. XI, p. 228]). In case $\mathfrak{f}$ admits a nondegenerate adinvariant (possibly indefinite) scalar product $B$ (such as the Killing form for semisimple $\mathrm{f}$ ) the 0 -connection $\nabla$ is always equal to the Levi-Civita connection of the left and right invariant (pseudo) Riemannian metric on $K$ which is induced by $B$. Now, since $r(x)=1 / 2 \operatorname{ad}(x), x \in \mathfrak{f}$, the Lie algebra $\mathfrak{a}_{\mathfrak{f}}$ is equal to $\operatorname{ad}(\mathfrak{f})$ $\equiv\{\operatorname{ad}(x) \mid x \in \mathfrak{f}\}$ which is isomorphic to $\mathfrak{f}$ modulo its centre. Taking $\mathfrak{g}=\mathfrak{F}$ and $\varrho^{*}=$ ad one is in the situation that $\mathrm{g}$ is a Baxter Lie algebra (cf. Sect. 2) where $R$ is equal to 1 after rescaling with a factor of 2 (which of course satisfies the MCYBE [cf. Eq. (2.20)]). However, in the typical cases of the Belavin-Drinfel'd classification scheme (cf. [3]) the situation is not so trivial: $\mathfrak{g}$ is simple and $\mathfrak{f}$ is sometimes solvable, hence $g$ and $\mathfrak{f}$ are in general nonisomorphic. Therefore noncanonical left invariant affine symmetric structures on $K$ become important in this framework.

A more general reason why affine symmetric structures turn up can be derived using the concept of twilled extensions of two Lie algebras which is due to Kosmann-Schwarzbach and Magri (cf. [6]). In the theory of the above-mentioned Lie bialgebras the Lie algebras $\mathfrak{g}$ and $\mathfrak{g}^{*}=\mathfrak{f}$ can be "summed up" to a socalled double (cf. [5]) or Manin triple algebra $\mathfrak{g} \oplus \mathrm{g}^{*}$, where both $\mathrm{g}$ and $\mathrm{g}^{*}$ are (in general noncommuting) subalgebras of $\mathfrak{g} \oplus \mathfrak{g}^{*}$. More generally, the question arises under which circumstances a Lie structure can be defined on the direct vector space sum $\mathfrak{g} \oplus \mathfrak{f}$ of two arbitrary Lie algebras $\mathfrak{g}$ and $\mathfrak{f}$ such that both $\mathfrak{g}$ and $\mathfrak{f}$ are subalgebras with their original Lie structure. Kosmann-Schwarzbach and Magri called $\mathfrak{g} \oplus \mathfrak{f}$ a twilled extension of $\mathfrak{g}$ and $\mathfrak{f}$ and showed that a Lie structure on $\mathfrak{g} \oplus \mathfrak{f}$ in the above sense is equivalent to having the following ingredients (cf. [6]):

a)

b)

c)

d)

$$
\text { a representation } \varrho^{*}: \mathfrak{g} \rightarrow \mathfrak{g l}(\mathfrak{f}),
$$

$$
\text { a representation } \sigma: \mathfrak{f} \rightarrow \mathfrak{g l}(\mathfrak{g}) \text { such that }
$$

$$
\begin{aligned}
\varrho^{*}(\xi)[x, y]_{R}= & {\left[\varrho^{*}(\xi) x, y\right]+\left[x, \varrho^{*}(\xi) y\right]-\varrho^{*}(\sigma(x) \xi) y+\varrho^{*}(\sigma(y) \xi) x, } \\
\sigma(x)[\xi, \eta]= & {[\sigma(x) \xi, \eta]+[\xi, \sigma(x) \eta]-\sigma\left(\varrho^{*}(\xi) x\right) \eta+\sigma\left(\varrho^{*}(\eta) x\right) \xi } \\
& \text { for all } \xi, \eta \in \mathfrak{g} \text { and } x, y \in \mathfrak{f} .
\end{aligned}
$$

Then a Lie bracket on $\mathfrak{g} \oplus \mathfrak{f}$ can be defined as follows:

$$
[(\xi, x),(\eta, y)] \equiv\left([\xi, \eta]+\sigma(x) \eta-\sigma(y) \xi,[x, y]_{R}+\varrho^{*}(\xi) y-\varrho^{*}(\eta) x\right)
$$

and conditions a)-d) guarantee the Jacobi identity of this bracket.

In the situation of Sect. 2 we are already given two Lie algebras $g$ and $f$ and a representation $\varrho^{*}$ but we are still lacking a second representation $\sigma$. To get an idea we calculate condition c) above:

$$
\begin{aligned}
\xi & {[x, y]_{R}-[\xi \cdot x, y]_{R}-[x, \xi \cdot y]_{R} } \\
& =\xi \cdot(r(x) \cdot y)-\xi \cdot(r(y) \cdot x)-r(\xi \cdot x) \cdot y+r(y) \cdot(\xi \cdot x)-r(x) \cdot(\xi \cdot y)+r(\xi \cdot y) \cdot x \\
& =-[r(x), \xi] \cdot y-r(\xi \cdot x) \cdot y+[r(y), \xi] \cdot x+r(\xi \cdot y) \cdot x .
\end{aligned}
$$

In order to satisfy c) we can try to define the (would-be) representation $\sigma$ as follows:

$$
\sigma(x)(\xi) \equiv[r(x), \xi]+r(\xi \cdot x) \text { for all } \xi \in \mathfrak{g}, x \in \mathfrak{f} .
$$


With this ansatz we also check condition d) above:

$$
\begin{aligned}
\sigma(x) & ([\xi, \eta])-[\sigma(x)(\xi), \eta]-[\xi, \sigma(x)(\eta)] \\
= & {[r(x),[\xi, \eta]]+r([\xi, \eta] \cdot x)-[[r(x), \xi], \eta]-[r(\xi \cdot x), \eta] } \\
& -[\xi,[r(x), \eta]]-[\xi, r(\eta \cdot x)] \\
= & -[r(\xi \cdot x), \eta]-r(\eta \cdot(\xi \cdot x))+[r(\eta \cdot x), \xi]+r(\xi \cdot(\eta \cdot x)) \\
= & -\sigma(\xi \cdot x)(\eta)+\sigma(\eta \cdot x)(\xi)
\end{aligned}
$$

which is automatically satisfied by $\sigma$. The remaining check of the representation identity b) for $\sigma$ will become a little bit less trivial:

3.14. Theorem. We use the above notations. With the map $\sigma$ defined above (cf. Eq. (3.13)) the two Lie algebras $\mathfrak{g}$ and $\mathfrak{f}$ admit a twilled extension $\mathfrak{g} \oplus \mathfrak{f}$ iff the curvature tensor $R(x, y)=[r(x), r(y)]-r\left([x, y]_{R}\right)$ is $\mathfrak{g}$-invariant. In particular, the Lie group $K$ must be an affine locally symmetric space. In case $\mathrm{g}$ being generated by the $r$-matrices $\left(\mathfrak{g}=\mathfrak{a}_{\mathfrak{f}}, \varrho^{*}=\right.$ identity on $\left.\mathfrak{a}_{\mathfrak{t}}\right)$ the affine locally symmetric structure of $K$ is also sufficient to guarantee a twilled extension.

Proof. We calculate the representation identity b). Note that

$$
\sigma(x)(r(z))=R(x, z)+r(r(x) \cdot z) \quad \text { for all } x, z \in \mathfrak{f},
$$

and it follows that for all $x, y \in \mathfrak{f}$ and all $\xi \in \mathfrak{g}$,

$$
\begin{aligned}
\sigma(x) & (\sigma(y)(\xi))-\sigma(y)(\sigma(x)(\xi))-\sigma\left([x, y]_{R}\right)(\xi) \\
= & {[r(x),[r(y), \xi]]+r([r(y), \xi] \cdot x)+\sigma(x)(r(\xi \cdot y)) } \\
& -[r(y),[r(x), \xi]]-r([r(x), \xi] \cdot y)-\sigma(y)(r(\xi \cdot x)) \\
& -\left[r\left([x, y]_{R}\right), \xi\right]-r(\xi \cdot(r(x) \cdot y))+r(\xi \cdot(r(y) \cdot x)) \\
= & {[R(x, y), \xi]+r(r(y) \cdot(\xi \cdot x))+R(x, \xi \cdot y)+r(r(x) \cdot(\xi \cdot y)) } \\
& -r(r(x) \cdot(\xi \cdot y))-R(y, \xi \cdot x)-r(r(y) \cdot(\xi \cdot x)) \\
= & -([\xi, R(x, y)]-R(\xi \cdot x, y)-R(x, \xi \cdot y)),
\end{aligned}
$$

using Eq. (*) in the second equation. Since conditions a), c), and d) had already been shown to be valid this proves the first statement of the theorem. Now, setting $\xi$ equal to $r(z)=\left.\nabla_{z}\right|_{e}$ this clearly implies $\nabla R=0$ which is equivalent to the affine locally symmetric structure of $K$ (compare Theorem 3.7). On the other hand, since Eq. $(* *)$ defines a representation of all $\xi \in \mathfrak{g l}(\mathfrak{f})$ in the linear space of all bilinear antisymmetric maps: $\mathfrak{f} \times \mathfrak{f} \rightarrow \mathfrak{g l}(\mathfrak{f})$ then the vanishing of the right-hand side of Eq. (**) for all $\xi=r(z)=\left.\nabla_{z}\right|_{e}(\Leftrightarrow \nabla R=0)$ will imply the vanishing of the right-hand side of Eq. (**) for all commutator expressions formed out of the $r(z)$, i.e. for all $\xi \in \mathfrak{a}_{\mathfrak{k}}$. This proves the last statement of the theorem.

Now we see that Theorem 3.7 (and thus every (M)CYBE) is a special case of the above theorem since merely the $G$-invariance of the curvature tensor $-[c(x), c(y)]$ was important. Moreover, Theorem 3.14 allows us to form twilled extensions in all the cases covered by Theorem 2.18 (and contains the Manin triple algebras as a special case).

Kosmann-Schwarzbach and Magri (cf. [6]) gave a nice explanation for the funny form (3.13) of the representation $\sigma$ : considering $\sigma$ as a map $\mathfrak{g} \rightarrow \operatorname{Hom}(\mathfrak{f}, \mathfrak{g})$ : $\xi \mapsto(x \mapsto \sigma(x)(\xi))$ and the space of linear maps $\mathfrak{f} \rightarrow \mathfrak{g}, \operatorname{Hom}(\mathfrak{f}, \mathfrak{g})$, as a $\mathfrak{g}$-module (for 
$s \in \operatorname{Hom}(\mathfrak{f}, \mathfrak{g}) \operatorname{set}(\xi \cdot s)(x) \equiv[\xi, s(x)]-s(\xi \cdot x))$, condition (3.11) for $\sigma$ then says that $\sigma \in \Lambda^{1}(\mathfrak{g}, \operatorname{Hom}(\mathfrak{f}, \mathfrak{g}))$ is a cocycle. In particular, if $\sigma$ is a coboundary it will take the form (3.13). For instance, this happens for those Lie algebras whose first cohomology group $H^{1}(\mathfrak{g}, \operatorname{Hom}(\mathfrak{f}, \mathfrak{g})$ ) vanishes (which is true for all semisimple Lie algebras due to the first Whitehead lemma).

We remark that the Lie algebra $a_{\mathfrak{t}}$ was introduced by Kostant (cf. [15, Chap. X, p. 207]) and contains the Lie algebra of the holonomy group at the identity (which is generated by all parallel transports along loops based at the identity, cf. [15, Chap. II, p. 71]) as an ideal (cf. [15, Chap. X, p. 207]).

For globally symmetric spaces, the following geometric picture may be useful: Let $A$ be the identity component of the Lie group of all affine transformations on $K$ and let $a$ its Lie algebra. Since the left multiplications of $K$ on itself are affine, $K$ can be considered as a subgroup of $A$ and $A$ acts transitively on $K$. Let $H$ be the closed subgroup of $A$ that fixes the identity and let $\mathfrak{h}$ be its Lie algebra. Then $K$ is diffeomorphic to the homogeneous space $A / H$ in a natural way and one has the decomposition $A=K H$ with $K \cap H=\{e\}$ because $K$ clearly acts freely and transitively on itself. This corresponds to a direct (vector space) sum $\mathfrak{a}=\mathfrak{h} \oplus \mathfrak{f}$. Now, in case $K$ is an affine symmetric space there is also a direct decomposition $\mathfrak{a}=\mathfrak{h} \oplus \mathfrak{m}$, where $\mathfrak{m}$ is a vector subspace of $\mathfrak{a}$ and the following well-known commutation relations hold: $[\mathfrak{h}, \mathfrak{m}] \subset \mathfrak{m}$ and $[\mathfrak{m}, \mathfrak{m}] \subset \mathfrak{h}$ (cf. [15, Chap. XI, p. 226]). For $a \in \mathfrak{a}$ let $a_{\mathfrak{h}}$ (respectively $a_{\mathfrak{m}}$ ) denote the $\mathfrak{h}$ - (respectively $\mathfrak{m}$-) components of $a$ according to the decomposition $\mathfrak{a}=\mathfrak{h} \oplus \mathfrak{m}$. Similarly, let $\pi_{\mathfrak{h}} a$ (respectively $\pi_{\mathfrak{t}} a$ ) denote the $\mathfrak{h}$ - (respectively $\mathfrak{f}$-) components of $a$ according to the decomposition $\mathfrak{a}=\mathfrak{h} \oplus \mathfrak{f}$. Now, every symmetric space always carries the so-called canonical connection (which is in general different from the above mentioned canonical affine symmetric structure), cf. [15, Chap. XI, p. 230]. It is usually expressed in terms of $\mathfrak{h}$ and $\mathfrak{m}$, where the tangent space $T_{e} K$ is identified with the subspace $\mathfrak{m}:\left(\nabla_{\hat{x}} \hat{y}(e)\right)_{\mathfrak{m}}$ $=(\Lambda(x)(y))_{\mathfrak{m}}=\left[x_{\mathfrak{h}}, y_{\mathfrak{m}}\right]+\Lambda_{\mathfrak{m}}\left(x_{\mathfrak{m}}\right)\left(y_{\mathfrak{m}}\right)=\left[x_{\mathfrak{h}}, y_{\mathfrak{m}}\right]$, since $\Lambda_{\mathfrak{m}}$ is equal to zero for symmetric spaces (cf. [15, Chap. X, p. 191], [Eq. (4.4)] of this paper, [15, Chap. X, p. 188, and Chap. XI, p. 230]). Note that $\pi_{\mathfrak{f}}$ is a bijection $m \rightarrow \mathfrak{f}$ which is the inverse of ()$_{\mathfrak{m}}: \mathfrak{i} \rightarrow \mathfrak{m}$, so if we map $\left(\nabla_{\hat{x}} \hat{y}(e)\right)_{\mathfrak{m}}$ from $\mathfrak{m}$ back to ${ }^{\prime}$ we get

$$
\nabla_{\hat{x}} \hat{y}(e)=r(x) \cdot y=\pi_{\mathfrak{t}}\left[x_{\mathfrak{h}}, y\right] \text { for all } x, y \in \mathfrak{f},
$$

where the commutator $[$,$] is taken in \mathfrak{a}$. Note that the commutation relation $[\mathrm{m}, \mathrm{m}] \subset \mathfrak{h}$ is crucial for the equation $[x, y]_{R}=r(x) \cdot y-r(y) \cdot x$. Moreover, the curvature tensor is easily calculated to be the usual one: $R(x, y) z=-\pi_{\mathfrak{t}}\left[\left[x_{\mathfrak{m}}, y_{\mathfrak{m}}\right]\right.$, $\left.z_{\mathfrak{m}}\right]$ for all $x, y, z \in \mathfrak{f}$ (cf. [15, Chap. XI, p. 231]). Now, define $\mathfrak{g}=\mathfrak{h}$ and

$$
\varrho^{*}(\xi) x=\pi_{\mathfrak{t}}[\xi, x] \text { for all } x \in \mathfrak{f}, \xi \in \mathfrak{g} .
$$

Hence $r(x)=x_{\mathfrak{h}}$ is a consistent choice by Eq. (3.15). Calculating for $x \in \mathfrak{f}, \xi \in \mathfrak{h}$,

$$
\begin{aligned}
\sigma(x)(\xi) & =[r(x), \xi]+r(\xi \cdot x)=\left[x_{\mathfrak{h}}, \xi\right]+\left(\pi_{\mathfrak{h}}[\xi, x]\right)_{\mathfrak{h}}=\left[x_{\mathfrak{h}}, \xi\right]+([\xi, x])_{\mathfrak{h}}-\left(\pi_{\mathfrak{h}}[\xi, x]\right)_{\mathfrak{h}} \\
& =\left[x_{\mathfrak{h}}, \xi\right]-[x, \xi]_{\mathfrak{h}}-\pi_{\mathfrak{h}}[\xi, x]=0+\pi_{\mathfrak{h}}[x, \xi],
\end{aligned}
$$

one gets the following equation for the representation $\sigma$ :

$$
\sigma(x)(\xi)=\pi_{\mathfrak{h}}[x, \xi] \quad \text { for all } x \in \mathfrak{f}, \xi \in \mathfrak{g} .
$$

Comparing these two equations with the bracket Eq. (3.12) one sees that the twilled extension of $\mathfrak{g}$ and $\mathfrak{f}$ is indeed $\mathfrak{a}$. This is also reflected by the geometrical fact that for symmetric spaces every parallel transport can be expressed by the action of 
an affine transformation (cf. [15, Chap. VI, p. 262]). Since the Lie algebra $\mathfrak{a}_{\mathfrak{f}}$ generates parallel transports along one-parameter-subgroups it is therefore contained in the Lie algebra of all infinitesimal affine transformations.

It should also be mentioned that representations like Eqs. (3.16) and (3.17) occur in the generalized Lax pairs discussed by Semenov-Tyan-Shanskii (cf. $[5,10])$ where also the Poisson commutativity ansatz of Adler/Kostant/Symes (cf. e.g. [7]) is applied.

As an example, consider $A=S L(N, \mathbb{R}), H=S O(n)$ and $K$ the subgroup of $A$ consisting of all upper triangular matrices with positive diagonal entries. Clearly, $H \cap K=\{1\}$. By the Iwasawa decomposition of $S L(n, \mathbb{R})$ (compare [17, Chap. VI, p. 260 and p. 270], and take inverse matrices) one has $A=K H$. Hence $K \sim A / H$ $=S L(n, \mathbb{R}) / S O(n)$. Now the homogeneous space $A / H$ is known to be isomorphic to the Riemannian symmetric space of all positive definite matrices of determinant one (cf. [17, Chap. X, p. 518]). Hence the Lie group $K$ is isomorphic to this space and thus carries a left invariant Riemannian symmetric structure. The projection ()$_{\mathfrak{b}}$ (respectively ()$\left._{\mathfrak{m}}\right)$ is the usual projection on the antisymmetric (respectively symmetric) part of a traceless matrix and the projection $\pi_{\mathfrak{h}}$ (respectively $\pi_{\mathfrak{f}}$ ) is easily computed to be $\pi_{\mathfrak{h}} a=a_{-}-a_{-}^{t}$ (respectively $\pi_{\mathfrak{f}} a=a_{0}+a_{+}+a_{-}^{t}$ ) where $a_{0}$ (respectively $a_{-}$, respectively $a_{+}$) denotes the diagonal (respectively strictly lower diagonal, respectively strictly upper diagonal) part of $a \in \mathfrak{a}$ and ( $)^{t}$ denotes matrix transposition. Therefore, by Eq. (3.15)

$$
r(x) \cdot y=1 / 2[x, y]-1 / 2 \pi_{\mathfrak{f}}\left[x^{t}, y\right] \text { for all } x, y \in \mathfrak{f},
$$

which is for $n \geqq 2$ noncanonical.

\section{Left Invariant Geodesic Motion on a Lie Group: Non(co)adjoint Lax Pairs}

In this section we shall combine the results of the two former parts to get an example of a nontrivial Lax pair. Let $K$ be a connected simply connected Lie group with Lie algebra $\mathfrak{f}$. We recall the basic procedure of building up Hamiltonian mechanics on $K$ as configuration space (for details see [13, 18]). The corresponding phase space is then the cotangent bundle $T^{*} K$ of $K$. We use left trivialization to get the diffeomorphism $T K \sim K \times \mathfrak{f}$, namely $v_{k} \mapsto\left(k,\left(T_{e} L_{k}\right)^{-1} v_{k}\right)$ for $k \in K$ and $v_{k} \in T_{k} K$. Similarly, we get the diffeomorphism $T^{*} K \sim K \times \mathfrak{f}^{*}$, namely $\beta_{k} \mapsto\left(k,\left(T_{e} L_{k}\right)^{*} \beta_{k}\right)$ for $k \in K, \beta_{k} \in T_{k} K^{*}$. By a second left trivialization step we get the diffeomorphism $T T^{*} K \sim K \times \mathfrak{l}^{*} \times \mathfrak{l} \times \mathfrak{l}^{*}$. The canonical 1 -form $\vartheta_{0}$ is given by $\vartheta_{0}(k, \beta)(k, \beta, x, \gamma)=\beta(x)$ and the canonical symplectic form $\omega_{0}=-d \vartheta_{0}$ is computed to be $\omega_{0}(k, \beta)\left(\left(k, \beta, x_{1}, \gamma_{1}\right),\left(k, \beta, x_{2}, \gamma_{2}\right)\right)=\gamma_{2}\left(x_{1}\right)-\gamma_{1}\left(x_{2}\right)+\beta\left(\left[x_{1}, x_{2}\right]_{R}\right)$ for $k \in K, x$, $x_{1}, x_{2} \in \mathfrak{I}, \beta, \gamma, \gamma_{1}, \gamma_{2} \in \mathfrak{I}^{*}$. The canonical point transformations $T^{*} L_{k}$ induced by the left multiplications $L_{k}, k \in K$, are simply given as follows: $T^{*} L_{k}\left(k^{\prime}, \beta\right)=\left(k k^{\prime}, \beta\right)$. Hence every left invariant Hamiltonian function is a real valued smooth function $F$ of the dual space $\mathfrak{I}^{*}$ alone, its differential $d F(\beta), \beta \in \mathfrak{I}^{*}$, lies in $\mathfrak{f}$ and the Hamiltonian equations of motion take the following form (see also [18]):

$$
\begin{aligned}
& \dot{k}=T_{e} L_{k} d F(\beta), \\
& \dot{\beta}=-\operatorname{ad}_{\mathfrak{t}}^{*}(d F(\beta))(\beta) .
\end{aligned}
$$

For matrix groups the first equation reads $\dot{k}=k d F(\beta)$. The second equation, also known as the Euler-Arnol'd equation, is independent of $k$ and has the well-known 
coadjoint Lax form: $\mathfrak{g} \equiv \mathfrak{f}, V \equiv \mathfrak{f}^{*}, \varrho \equiv \mathrm{ad}_{\mathrm{f}}^{*}, L(k, \beta) \equiv \beta$ and $M(k, \beta) \equiv d F(\beta)$. A special case of this is Euler's equation for a free top, where $K$ is the rotation group in $\mathbb{R}^{3}, L$ is the angular momentum in a body-fixed frame and $M$ is the angular velocity (in the same frame) that depends on $L$ via the moments of inertia. Moreover, the $V$-valued function $L$ satisfies the following Poisson bracket relation:

$$
\{\langle L, x\rangle,\langle L, y\rangle\}=-\left\langle L,[x, y]_{R}\right\rangle,
$$

which can directly be calculated using the above symplectic form or by noting that $L: T^{*} K \rightarrow \mathfrak{I}^{*}$ is the canonical momentum map corresponding to the canonical point transformations induced by right multiplication of $K$ on itself (see [13, Chap. 4.4, p. $311 \mathrm{ff}]$ ).

Now, let $Q$ be a nondegenerate symmetric bilinear form on the Lie algebra f. $Q$ extends to a left invariant pseudo-Riemannian metric on $K: Q(k)((k, x),(k, y))$ $\equiv Q(x, y)$ for all $x, y \in \mathfrak{f}$. Let $Q^{*}: \mathfrak{f}^{*} \times \mathfrak{F}^{*} \rightarrow \mathbb{R}$ denote the inverse metric. Then it is known (cf. $\left[13\right.$, p. 224]) that the function $F: \mathfrak{f}_{-}^{*} \rightarrow \mathbb{R}$,

$$
F \circ L(k, \beta) \equiv F(\beta) \equiv 1 / 2 Q^{*}(\beta, \beta) \quad \text { for all } \beta \in \mathfrak{f}^{*}
$$

defines a left invariant kinetic energy function $F \circ L$ on $T^{*} K$ whose Hamiltonian flow lines project onto geodesics in $K$. Let $\nabla$ be the Levi-Civita connection of $Q$. Then $\nabla$ is torsion-free and also left invariant and gives rise to an $r$-matrix as described in the previous part: $r(x) y=\nabla_{x} \hat{y}(e)$ for all $x, y \in \mathfrak{f}$. Set $\mathfrak{g}$ equal to $\mathfrak{a}_{\mathfrak{t}}$ [cf. Eq. (3.4)], let $\varrho^{*}$ be the natural representation of $\mathfrak{g} \subset \mathfrak{g l}(\mathfrak{f})$ acting as linear maps on $\mathfrak{f}$, set $V \equiv \mathfrak{f}^{*}$ and $\varrho \equiv\left(\varrho^{*}\right)^{*}$. For $x \in \mathfrak{f}$, let $x_{K}$ denote the vector field $k \mapsto \frac{d}{d t}$ $\left.((\exp t x) k)\right|_{t=0}$, i.e. the infinitesimal generator of the left multiplication of $K$ on itself. Note that $x_{K}$ is a right invariant vector field on $K$ and obeys the equation $x_{K}(k)=\left(\operatorname{Ad}\left(k^{-1}\right) x\right)^{\wedge}(k)$. Hence, it can be shown that for all $x, y \in \mathfrak{f}$ the following relation holds (see also [15, Chap. X, p. 188]):

$$
r(x) y=\left(\nabla_{x_{K}} y_{K}-\left[x_{K}, y_{K}\right]\right)(e) \text {, }
$$

where $[$,$] denotes the Lie bracket of vector fields. Since all the x_{K}$ are Killing fields for the metric $Q$ (i.e. $L_{x_{K}} Q=0, L$ denoting the Lie derivative here) and $Q$ is covariantly constant (i.e. $\nabla Q=0$ ) it can be deduced that all the linear maps $r(x)$, $x \in \mathfrak{f}$, are $Q$-antisymmetric, i.e.

$$
Q(r(x) y, z)+Q(y, r(x) z)=0 \text { for all } x, y, z \in \mathfrak{f}
$$

(cf. also [15, Chap. X, p. 201/202]). Since the $r(x), x \in \mathfrak{f}$, generate the Lie algebra $\mathfrak{g}=\mathfrak{a}_{\mathfrak{t}}$ and the previous equation defines a representation of $\mathfrak{g l}(\mathfrak{f})$ on the space of all symmetric bilinear forms on $\mathfrak{f}$ one has the following equation, too:

$$
Q(\xi y, z)+Q(y, \xi z)=0 \text { for all } y, z \in \mathfrak{f}, \xi \in \mathfrak{g} .
$$

This easily implies that a similar equation holds for the inverse metric $Q^{*}$ :

$$
Q^{*}(\varrho(\xi) \beta, \gamma)+Q^{*}(\beta, \varrho(\xi) \gamma)=0 \text { for all } \beta, \gamma \in \mathfrak{f}^{*}, \xi \in \mathfrak{g} .
$$

Specializing to $\beta=\gamma$ the above equation simply says that the function $F$ representing the kinetic energy function [cf. Eq. (4.3)] is infinitesimally $G$-invariant:

$$
d F(\beta) \varrho(\xi) \beta=0 \text { for all } \beta \in \mathfrak{f}^{*}, \xi \in \mathfrak{g} .
$$


But according to Eq. (2.15) this means that the kinetic Hamiltonian $F \circ L$ admits the following second Lax pair:

$$
\dot{L}=\varrho\left(r(d F(L)) L=\varrho\left(r\left(Q^{\#} L\right)\right) L,\right.
$$

where $\mathfrak{g} \equiv \mathfrak{a}_{\mathfrak{t}}, \varrho=1_{\mathfrak{g}}, V \equiv \mathfrak{l}^{*}, L(k, \beta) \equiv \beta, M(k, \beta) \equiv-r(d F(L(k, \beta)))=-r\left(Q^{\#} \beta\right)\left(Q^{\#}\right.$ being the inverse metric regarded as a linear map $\left.V=\mathfrak{f}^{*} \rightarrow \mathfrak{f}\right)$. If we rescale $L$ and $M$ by a factor of -1 we see that by the rescaled generalized Lax equation (4.9) and Eq. (4.2) the hypothesis of Theorem 3.6 is satisfied.

The first conclusion which can be drawn from this is the fact that each $\mathfrak{F}^{*}$-part $\beta(t)$ of the solution curve is contained in the intersection of some coadjoint orbit [according to the first Lax pair Eq. (4.1)] with some G-orbit (according to the second Lax pair Eq. (4.9)]. For the above-mentioned free top this picture corresponds to the well-known fact (cf. e.g. [13, p. 363]) that the $L$ curves lie in the intersection of a sphere (the (co)adjoint orbit of the usual Euclidean $S O(3)$ action) with an ellipsoid [the orbit of the group $G \subset S L(3, \mathbb{R})$ that leaves invariant the inverse inertia tensor]. Note that $G$ is isomorphic but not equal to $S O(3)$ in this example.

The second conclusion is that according to the discussion in the two previous parts all the functions of the form $f \circ L$, where $f: \mathfrak{f}^{*} \rightarrow \mathbb{R}$ is $G$-invariant Poisson commute among themselves and of course with the Hamiltonian. From the first Lax pair alone one would merely conclude that all the functions $f \circ L$, where $f: \mathfrak{F}^{*}$ $\rightarrow \mathbb{R}$ is $\mathrm{Ad}^{*}(K)$-invariant Poisson commute with the Hamiltonian and among themselves. Hence the additional $G$-symmetry produces new Poisson commuting conservation laws. Moreover, since each $\mathrm{Ad}^{*}$-invariant function of $L$ Poisson commutes with any function of $L$ (cf. e.g. [7]) one can put these two sets of functions together to get a bigger family of Poisson commuting conservation laws. However, it may happen that the group $G$ becomes as large as possible, namely isomorphic to $S O(p, q)((p, q)$ denoting the signature of $Q)$. Then the generic orbits of $G$ are manifolds of codimension one in $\mathfrak{f}^{*}$ and $F$ is (up to functional dependence) the only $G$-invariant function on $\mathfrak{f}^{*}$.

Acknowledgements. I would like to thank C. Devchand, T. Filk, M. Forger, K. Happle, J. Hoppe, M. Koch, A. Medina Perea, L. O'Raifeartaigh, H. Römer, and B. Scheffold for fruitful discussions on this subject. In particular, I want to express my gratitude to T. Filk who suggested to me to use two $r$-matrices $r_{+}$and $r_{-}$instead of one and M. Koch who pointed out to me $[6,10]$.

\section{References}

1. Sklyanin, E.K.: On complete integrability of the Landau-Lifschitz equation. Preprint LOMI, E-3-79, Leningrad, LOMI (1980)

2. Sklyanin, E.K.: The quantum inverse scattering method. Zap. Nauch. Sem. LOMI 95, 55-128 (1980)

3. Belavin, A.A., Drinfel'd, V.G.: Solutions of the classical Yang-Baxter equation for simple Lie algebras. Funct. Anal. Appl. 16, 159-180 (1982), 17, 220-221 (1983)

4. Drinfel'd, V.G.: Hamiltonian structures on Lie groups, Lie bialgebras, and the geometrical meaning of the Yang-Baxter equations. Soviet Math. Dokl. 27, 68-71 (1983)

5. Semenov-Tyan-Shanskii, M.A.: What is a classical $r$-matrix? Funct. Anal. Appl. 17, 259-272 (1983)

6. Kosmann-Schwarzbach, Y., Magri, F.: Poisson Lie groups and complete integrability. I. Drinfel'd bigebras, dual extensions and their canonical representations. Ann. Inst. Henri Poincaré 49, 433-460 (1988) 
7. Babelon, O., Viallet, C.-M.: Integrable models, Yang-Baxter equation, and quantum groups. Part I. Ref. S.I.S.S.A. 54 EP (May 89) (preprint Trieste)

8. Babelon, O., Viallet, C.-M.: Hamiltonian structures and Lax equations. Phys. Lett. B 237, 411-416 (22 March 1990)

9. Lax, P.D.: Integrals of nonlinear equations of evolution and solitary waves. Commun. Pure Appl. Math. 21, 467-490 (1968)

10. Semenov-Tyan-Shanskii, M.A.: Classical $r$-matrices and the method of orbits. Zap. Nauch. Sem. LOMI 123, 77-91 (1983)

11. Fomenko, A.T., Trofimov, V.V.: Integrable systems on Lie algebras and symmetric spaces. New York: Gordon and Breach 1988

12. Ferreira, L.A.: Integrability and symmetric spaces. I. The group manifold. II. the coset spaces. Int. J. Mod. Phys. A 4, 649-674 (1989), (part I) and 675-699 (part II)

13. Abraham, R., Marsden, J.E.: Foundations of mechanics. 2nd (ed.) Princeton, NJ: Benjamin/Cummings 1978

14. Fomenko, A.T., Mishchenko, A.S.: Generalized Liouville method of integration of Hamiltonian systems. Funct. Anal. Appl. 12, 113-121 (1978)

15. Kobayashi, S., Nomizu, K.: Foundations of differential geometry. New York: Wiley, Vol. I (1963) and Vol. II (1969)

16. Medina Perea, A.: Flat left invariant connections adapted to the automorphism structure of a Lie group. J. Diff. Geom. 16, 445-474 (1981)

17. Helgason, S.: Differential geometry, Lie groups, and symmetric spaces. New York: Academic Press 1978

18. Fomenko, A.T., Mishchenko, A.S.: Euler equations on finite-dimensional Lie groups. Math. USSR Izv. 12, 371-389 (1978)

Communicated by N. Ya. Reshetikhin 